\title{
Optimization of fMRI Analysis of Speech Areas in Pre- and Postoperative Diagnostics
}

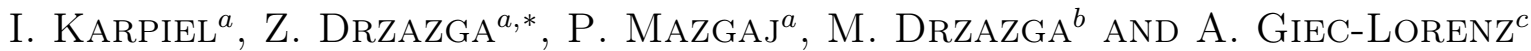 \\ ${ }^{a}$ Department of Medical Physics, Institute of Physics, University of Silesia, \\ Uniwersytecka 4, 40-007 Katowice, Poland \\ ${ }^{b}$ Institute of Computer Science, University of Silesia, Bedzinska 39, 41-200 Sosnowiec, Poland \\ ${ }^{c}$ Helimed Diagnostic Imaging Sp. z o.o., Laboratory of Magnetic Resonance Imaging, \\ Panewnicka 65, 40-760 Katowice, Poland
}

\begin{abstract}
Functional magnetic resonance imaging (fMRI) was used to characterize relevant functional areas adjacent to the tumor what has important implications for surgical intervention. The aim of our study was to evaluate the functional areas of Broca and Wernicke responsible for production and understanding the speech which are very important from the point of view patients quality life using different variants of analysis available in the SPM package. Brain activity imaging with FWE controlling seems to be more appropriate than this with un uncorrected thresholds in clinical diagnostic. However analysis in the SPM should be carried out with great care taking into consideration that results can be influenced by parameters used in statistical approaches.
\end{abstract}

DOI: $10.12693 /$ APhysPolA.133.728

PACS/topics: 87.57.Nk, 87.61.Pk, 87.66.Ur

\section{Introduction}

Functional magnetic resonance imaging (fMRI) utilizing the blood oxygenation level- dependent (BOLD) [1] contrast has become an important research and clinical tool to study the normal and diseased with tumor human brain. Neurosurgery in functionally relevant brain structures carries a high risk for surgery induced postoperative neurological deficits. For preoperative patients resection of brain tumours aims to remove as much of the changed tissue as possible while preserving essential brain functions simultaneously. Therefore, very important is the precise spatial relationship between the lesion and adjacent tissue (anatomical pathways) as well as include potential areas of activity brain. For this reason fMRI studies for localizing and areas of the human brain that govern e.g.: sensorimotor cortex, occipital, language or DMN (Default Mode Network) [2,3] are increasingly used in preoperative and postoperative diagnostics.

We focused on activations of speech areas - Broca linked with speech production and Wernicke responsible for understanding the speech because language areas are particularly important for patients. The aim of our study was to evaluate the functional areas located close to the lesions using different variants of analysis available in the SPM package.

Data acquired during course of fMRI require to simultaneously perform thousands of statistical tests to detect brain activity across a hundred thousand brain regions The need for appropriate multiple comparisons correction to quantify the like hood of obtained false positives

*corresponding author; e-mail: zofia.drzazga@us.edu.pl has been pointed out in many articles [4-6]. The most common metrics are the family-wise error rate (FWER), defined as the probability of obtaining at least one false positive in a family of tests, and the false discovery rate (FDR), defined as the proportion of false positive among all rejected tests. Either the FWER controlling procedure or FDR and also both together can be involved in analysis. Bright discussion regarding the appropriate multiple comparisons correction in the analysis of large data sets such as genetics, imaging and economic is served in [5]. In this paper an impact of some aspects of statistical analysis in practical research application of fMRI in pre- and postoperative diagnostic is presented.

\section{Materials and methods}

\subsection{Subjects and fMRI Data Acquisition}

Three preoperative patients with tumors (meningioma) and five postoperative (3 with astrocytoma (WHO II-III) and 2 with meningioma) were included in the study, comprising 3 females (a mean age $20.7 \pm 3.8$ ) and 5 males (a mean age $35 \pm 7.4$ ). Scanning was performed at the Helimed Diagnostic Imaging Center in Katowice as part of normal clinical work using a $1.5 \mathrm{~T}$ MAGENTOM Aera scanner (Siemens, Erlangen, Germany), equipped with a 20 -channel head-neck coil. The protocol included: 1) Ultrafast Gradient Echo 3D sequence (3D $\mathrm{T}_{1}$-MPRAGE, resolution $1 \times 1 \times 1 \mathrm{~mm}^{3}$, $\mathrm{TE} / \mathrm{TR}=2.67 \mathrm{~ms} / 1900 \mathrm{~ms}, \mathrm{TI}=1100 \mathrm{~ms}$, slice thickness $1.0 \mathrm{~mm}, \mathrm{FOV}=250 \times 250 \mathrm{~mm}^{2}$, matrix size $256 \times 256,192$ axial slices; 2) fMRI images (EPI SE sequence, resolution $3 \times 3 \times 3 \mathrm{~mm}^{3}, \mathrm{TE} / \mathrm{TR}=50 \mathrm{~ms} / 3140, \mathrm{TI}=1100 \mathrm{~ms}$, slice thickness $4.0 \mathrm{~mm}, \mathrm{FOV}=1320 \times 1320 \mathrm{~mm}^{2}$, matrix size $384 \times 384$, 36 axial slices per volume, covering both hemispheres). 


\subsection{Experimental paradigm}

In this post- and preoperative study we applied the same 2 paradigms. All paradigms to stimulate Wernicke and Broca areas were created and controlled by using the PsychoPy software program [7]. The software provides real time stimulus presentation, and accurate synchronization with the MR imager. Paradigms with classic blocked design composed of 5 active and 6 rest blocks collected 110 volumes. The first paradigm (I) consisted of associating activities with a displayed noun e.g. a chairsit. The second paradigm (II) was to generate words beginning with the letter (A-Anna, alligator,...).

\subsection{Data analysis}

Before data analysis, the DICOM format was transformed into other formats Neuroimaging Informatics Technology Initiative (NIfTI) file format [8]. Next steps was 'manual coregistration' which was the point 0 setting in Anterior Commissure (AC). The commissure has become key landmarks, because Talairach chose them to determine the standard alignment of the brain for their famous atlas. To align a scan Talairach space, a brain has to be set so that the anterior commissure (AC) and posterior commissure (PC) are on a horizontal line. It is relatively easy to see on most structural scans. All the preprocessing were carried out using the statistical parametric mapping SPM12 (). The pre- and postoperative imaging time series of each subject was 'realigned', 'slice timing', 'coregistration', 'segmentation', 'normalization' and 'spatial smoothing'. All images were spatially normalized into the Montreal Neurological Institute (MNI) [9]. Moreover analysis using mask allowing for show activations in areas that expected was performed. To prepare mask images of speech areas (Broca and Wernicke) used WFU Pickatlas for SPM. Mask was generated for both hemispheres. Localization of active areas were made using functional atlases and $[10,11]$ and Talairach Client [12].

In SPM analysis the approach of spatial smoothing is commonly used and is justified by the fact that fMRI data inherently show spatial correlations due to functional similarities of adjacent brain regions and the blurring of the vascular system [13]. Our statistical images were performed for fixed Gaussian kernels with width FWHM of $6 \mathrm{~mm}$ and extent threshold 0 voxels. These parameters were applied because provided the most sensible results as discussed in [14].

\section{Results and discussion}

Representative results of optimization of fMRI analysis of language areas in the studied patients are shown in Figs. 1 and 2 and Table I. Areas involved in language comprehension and production centred around the sylvian fissure of the dominant cerebral hemisphere, usually referred to as Broca (BA44, BA45) areas and Wernicke (BA 22) area could be found.
Figure 1 displays two different approaches Wernicke's activations in the same brain plane. After spatial normalization and manual coregistration of the point 0 into AC Wernicke area signal intensity is higher and localized in another coordinates than before and artifacts disappear in the postoperative box after resection astrocytoma (WHO III) in left temporal lobe. It follows from our studies that special attention should be paid to the manual coregistration method and normalization (which are rarely used in clinical practice) for diseased brain with tumor because these procedures can enhance the reliability and repeatability of studies $[9,15]$.

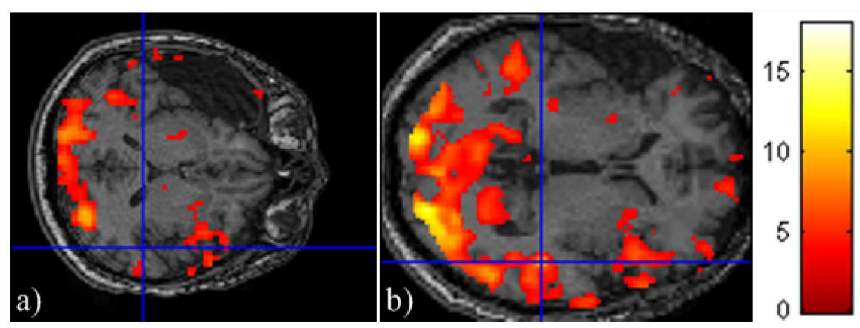

Fig. 1. Images of activations on Wernicke significance level $p<0.001$ for extent threshold 0 voxels. a) point 0 no setting in $\mathrm{AC}, \mathrm{b}$ ) position $\mathrm{AC}$ set in structural and functional images. Significance level $p<0.001$ for extent threshold 0 voxels.

Figure 2 shows comparison of images of Wernicke activity in representative preoperative patient obtained when the $T$-maps were performed using an uncorrected threshold $(p<0.0001$ and 0.001$)$ and with threshold designed to the FWE controlling procedure $(p<0.0001$ and 0.00005 ) in form of glass brain (Fig. 2a) and 2c)) as well as anatomical coronal slice (Fig. 2b) One can see large irregularly, oval shaped area in left lobus parietalis diagnosed as atypical meningioma which can induce relocation or disappearance of active areas (Fig. 2b). Moreover it is clearly that the choice of threshold value has a marked impact on which voxels are deemed active. The use of FEW controlling procedure allows to locate brain activity more accurately. In so-called glass brain (see Fig. 2a) all activations (from whole brain) that exceed the specified significance level are drawn. Analysis with FWE (0.00005) seems to be the most approciate for presented imaging cases of activation on Wernicke area. The another alternative imaging setting in focusing analysis to certain pre-specified region of interest could be performed what is illustrated in Fig. 2c. This substantially reduce the burden of multiple comparison correction by limiting the number of voxels of interest but there is the risk of "data dredging" and uncovering biased results that will not be reproducible. However use of masking as well as normalization in patients with lesions is still controversial and is the subject of much work lately [9, 15].

Comparison of impact of FWE controlling procedure on the amount of activations for statistic images obtained with Gaussian kernel widths (FWHM of 6) and extent threshold 0 voxels is listed in Table I. It should be noted 


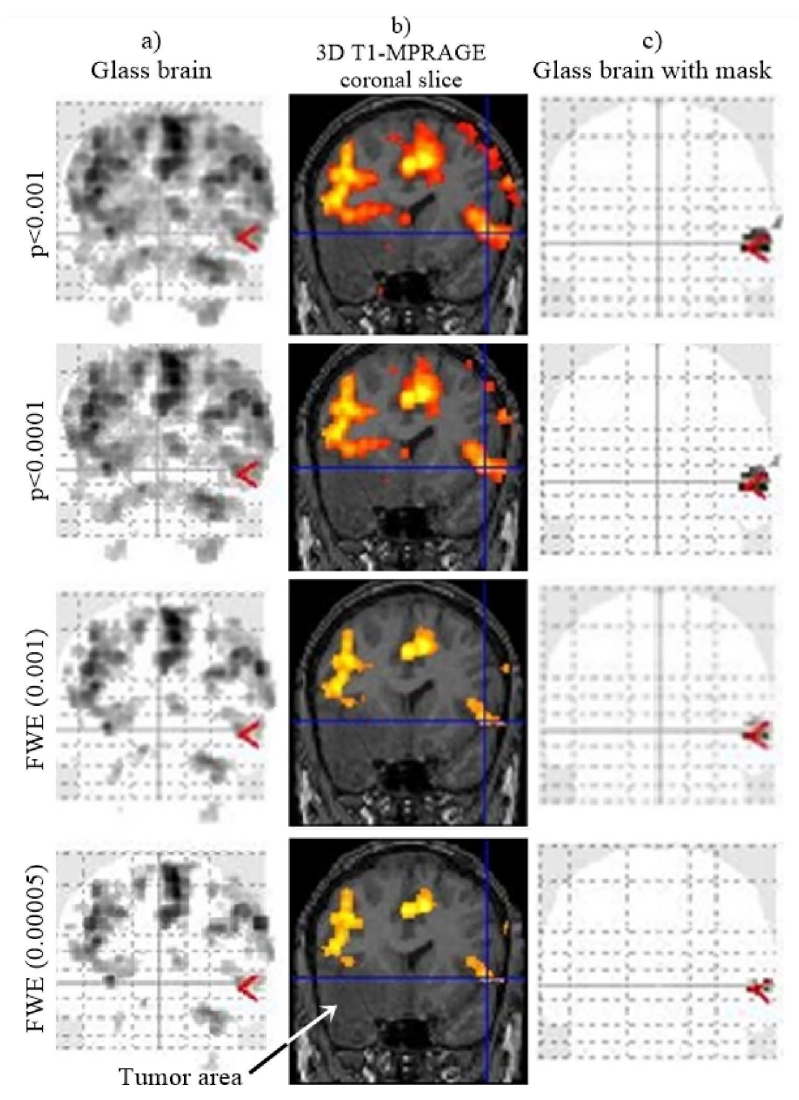

Fig. 2. Imaging of activations on Wernicke area obtained for kernel 6 significance level $p<0.001, p<$ $0.0001, p<0.00005$ for 0 voxels. Activations presented on a) glass brain, b) anatomy coronal slice and c) with Wernicke mask. After the manual coregistration (set point in point $\mathrm{AC}$ ) and normalization.

TABLE I

The obtained activations of brain in BA44, BA45 and BA 22 areas for both hemispheres (left (L) and right $(\mathrm{P})$ ) for paradigm II in the studied postoperative patients for significance level $p<0.001$ where $+/$ - means activity $/$ no activity and $\oplus$ indicates dominant hemisphere.

\begin{tabular}{|c|c|c|c|c|c|c|c|c|}
\hline & \multicolumn{4}{|c|}{ no correction } & \multicolumn{4}{|c|}{ FWE correction } \\
\hline & \multicolumn{2}{|c|}{ Wernicke } & \multicolumn{2}{|c|}{ Broca } & \multicolumn{2}{|c|}{ Wernicke } & \multicolumn{2}{|c|}{ Broca } \\
\hline & $\mathrm{L}$ & $\mathrm{P}$ & $\mathrm{L}$ & $\mathrm{P}$ & $\mathrm{L}$ & $\mathrm{P}$ & $\mathrm{L}$ & $\mathrm{P}$ \\
\hline 1 & $\oplus$ & + & + & $\oplus$ & $\oplus$ & - & + & $\oplus$ \\
\hline 2 & + & $\oplus$ & - & $\oplus$ & - & + & - & + \\
\hline 3 & $\oplus$ & + & $\oplus$ & + & $\oplus$ & - & $\oplus$ & - \\
\hline 4 & - & $\oplus$ & - & - & - & - & - & - \\
\hline 5 & $\oplus$ & + & $\oplus$ & - & - & - & + & - \\
\hline
\end{tabular}

that some results adjustment to control FWE do not reveal all brain activities that are clearly visible performing T- maps with a reasonable uncorrected threshold (e.g., $p<0.001)$. This means that analysis in the SPM should be carried out with great care in view of the diagnostic purpose.

\section{Conclusions}

fMRI method is a proper tool to assist with troubleshooting pre- and postoperative diagnostics which can explain neurological symptoms diseases of patients (e.g. disorders in the meaning of speech) and allows to plan surgery in order to reduce as much as possible neurological damage and next to monitor the postoperative patients. An appreciate selection of parameters in the analysis of SPM allows increase of diagnostic value of fMRI studies.

\section{References}

[1] S. Ogawa, T.M. Lee, A.S. Nayak, P. Glynn, Magnetic) Resonance in Medicine 14, 68 (1990.

[2] M. Tozakidou, H. Wenz, J. Reinhardt, E. Nennig, K. Riffel, M. Blatow, Ch. Stippich, Neuroimage: Clinical 2, 221 (2013).

[3] S. Marion, European Association of Neuro Oncology Magazine 2.3, 123 (2012).

[4] T. Nichols, S. Hayasaka, Statistical methods in medical research 12, 419 (2003).

[5] M.A. Lindquist, A. Mejia, Psyhosom Med 77, 114 (2015).

[6] M.A. Lindquist, Statistical Science 23, 439 (2008).

[7] J. W. Peirce, J. Neurosci. Methods 15, 162, 8 (2007).

[8] R. Cox, J. Ashburner, H. Breman, K. Fissell, C. Haselgrove, C. Holmes, J. Lancaster, D. Rex, S. Smith, J. Woodward, S. Strother, 10th Annual Meeting of the Organization for Human Brain Mapping (OHBM 2004) 2004.

[9] J. Crinion, J. Ashburner, A. Leff, M. Brett, C.Price, K. Friston, NeuroImage 37, 866 (2007).

[10] J. Tamraz, Y. Comair. Atlas of Regional Anatomy of the Brain Using MRI: With Functional Correlations, Springer, 2005.

[11] W. Orrison, Jr. Atlas funkcjonalny mózgu, PZWL, Warszawa 2010, (in Polish).

[12] Talairach.com, Talairach Client, Version 2.4.3.

[13] T. White, D. O'Leary, V. Magnotta, S. Arndt, M. Flaum, N.C. Andreasen, NeuroImage 13, 577 (2001).

[14] I. Karpiel, Z. Drzazga, P. Mazgaj, P. Ulrych, A.GiecLorenc, Innovations in Biomedical Engineering. Advances in Intelligent Systems and Computing, vol. 526, Eds.: M. Gzik, E. Tkacz Z. Paszenda E. Piętka, Springer 2016, p. 219.

[15] S.M. Andersen, S.Z. Rapcsak, P.M. Beeson, NeuroImage 53, 78 (2010). 